\title{
A Study on the Integrations of Products and Manufacturing Engineering using sensors and IoT
}

\author{
Dahye Hwang ${ }^{10000-0002-0990-1484]}$ and Sang Do Noh ${ }^{1 *}$ \\ ${ }^{1}$ Sungkyunkwan University, Suwon, South Korea \\ sdnoh@skku.edu
}

\begin{abstract}
In recent years, studies on smart manufacturing using ICT have increased significantly, and much attention has been paid to CPSs, IoT, sensors, industrial data analytics and artificial intelligence as core technologies. In particular, CPSs are one of the core technical elements in smart manufacturing, and a variety of studies on CPSs are underway. Accordingly, a large number of technical developments and applications related to intelligent and autonomous facilities, the prediction of factory operation, machinery factories and quality issues and proactive responses have been made. The new paradigm in smart manufacturing can be defined as customisation, connectivity and collaboration. The goal of smart manufacturing is to perform right decision making autonomously by connecting intelligent design, the efficient manufacture of customized products in accordance with the analysis of market and customer demand, sales, the user's use and the service sector. The paper presents the concept, framework, configuration and implementation method of a CPS that is performed intelligently based on the IoT, smart sensors and industrial data analytics from manufacturing preparation and execution.
\end{abstract}

Keywords: Cyber-physical system (CPS), Internet of things (IoT), Data analytics, Smart manufacturing, Smart product

\section{Introduction}

Much attention has been paid to information and communication technology (ICT), which has been consistently advanced through the Fourth Industrial Revolution. Accordingly, cyber-physical systems (CPSs), big data, artificial intelligence and the Internet of Things (IoT) have arisen as the main technical innovations, which have changed existing industrial structures significantly [1].

Industrie 4.0 is a strategy to respond to changes in society, technology, the economy, the ecosystem and politics faced by manufacturing in Germany with all efforts by combining ICT capabilities. In South Korea, the Manufacturing Innovation 3.0 program has been underway nationally to build and promote smart factories where all business processes of planning, design, production, distribution and sales are integrated through ICT and custom-tailored products are manufactured with minimum cost and time [2]. A CPS is a core technology that configures and operates smart manufacturing. It is a system that monitors, controls and regulates changes in physical environments autonomously 
by combining network technology with physical elements, such as machinery and workers as well as the surrounding environments [3]. Accordingly, a variety of studies have been conducted on CPSs. In particular, a large number of technical developments and applications related to intelligent and autonomous facilities, the prediction of factory operation, machinery factory and quality issues and proactive responses have been made [4].

The study presents a framework that connects and integrates product and manufacturing through the CPS and introduces an application of the framework to a cosmetic company.

\section{Research background}

Smart manufacturing refers to a strategy that combines technologies complexly to connect and operate facilities and factories and operation systems with the CPS concept for personalized manufacturing and operation excellence. Personalized manufacturing aims to provide customized products and services for operation and individual consumers collaboratively by combining facilities, factories and manufacturing information with services. Operation excellence aims to improve productivity, quality and cost via optimal responses to various changes rapidly and intelligently by sharing and utilizing production information and knowledge in real time.

Smart manufacturing can be defined as customization, connectivity and collaboration [5]. Customization represents an attribute of the market or demand side, while connectivity and collaboration represent attributes of the supply side as all components and participants in the value chain are connected in real time.

\subsection{Product Design in Smart Manufacturing}

Research on smart product design. Erdenebayar-Urtnasan et al. proposed a parameter derivation methodology to derive the design parameters of a universal ear shell for a hearing aid [6]. To reduce its time and cost, their study derived design parameters, and customer usage and product information are inputted, and machine learning algorithm is applied to train the data.

Harvard business review defined three elements that compose smart products [7]. A smart connected product consists of physical components, smart components, such as sensors and a data repository, and connectivity components. The capabilities of a smart connected product can be defined by four improvements: monitoring, the autonomous function control, optimization and diagnosing.

A study called 'Designing for Manufacturing's Internet of Things' introduces a case study on a smart connected product with the example of Dell [8]. Dell attached radio frequency identification (RFID) tags to each of 4,000 trays in the manufacturing process to deliver assembly information on the products. Through this process, Dell was able to collect various pieces of information more accurately than before. However, 
limitations such as lack of standardization of networks and interfaces, the security of information leakage, the deficient infrastructure of IoT equipment exist.

A study entitled 'Enabling process mining on sensor data from smart products' applied a process-mining methodology to Philips smart feeding bottles to discover patterns of customer behavior and propose a utilization measure [9]. In the above study, the log data of users were analyzed via the process-mining methodology using temperature and gyro sensors in the smart feeding bottle, thereby dividing the process into preparation, feeding and aftercare and deriving detailed sub-processes. This process is expected to help product designers as well as provide services to customers.

Three main elements of smart products. In smart manufacturing, as the advancement of ICT has influenced products, control software has been embedded in products, which have become more intelligent and serviceable through connection to the Internet [10].

Smart products can record information, such as the movement paths of products, using embedded RFID and classify product information through unique product ID. Furthermore, smart products influence the surrounding environment and monitor the surrounding environment and their own status to exchange information. Smart connected products can be divided into three components: physical components, such as product design; smart components, such as sensors and embedded software; and connectivity components for external services for products and data exchange.

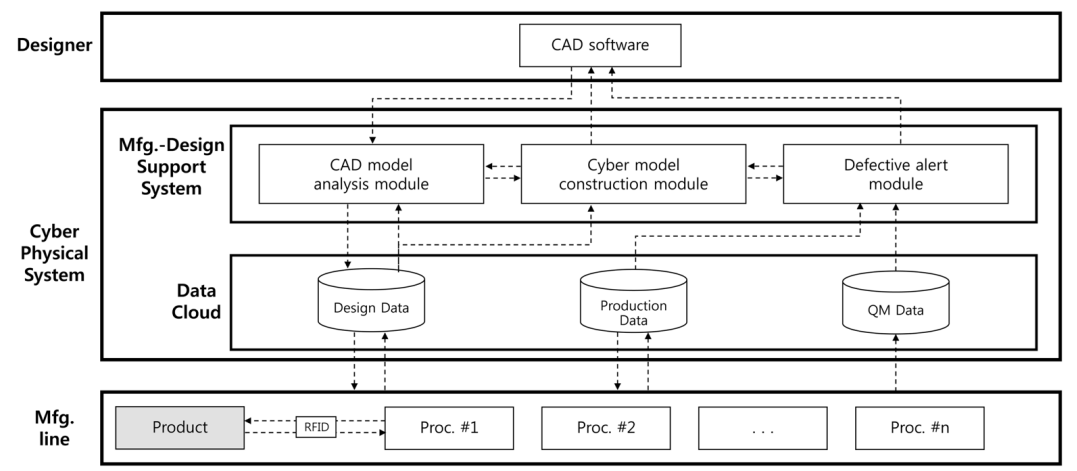

Fig. 1. Framework for product-manufacturing integration

\section{$3 \quad$ Integration framework for product and manufacturing}

The paper aims to design a CPS where the product and manufacturing are integrated. The basic framework including the product and manufacturing phase in the product life cycle is shown in Fig. 1, which shows activities and data flows. Collected information is stored on a cloud in an integrated manner, and feedback is given to product designers utilizing the collected information. 
In the paper, it is assumed that facilities with IoT sensors and database storing product process information are supporting data delivery among products, production facilities and processes through product IDs. In the production phase of the framework, a module that utilizes data obtained during manufacturing is designed, which plays a role as middleware that connects designers and production data.

In the production phase, design information and production information on the product is shared and utilized through communications between the product and facility using RFID [11]. To achieve this, an RFID tag is attached to every product while an RFID reader is attached to every facility. The production information on the product is fetched from the cloud by tagging the RFID of the product via the facility reader before the process starts. After a process is performed according to the production information, actual processing information and quality information on the product are collected and transferred to the cloud again.

If a defect occurs during the process, the ID of the defect is tagged, and up-to-date cumulative actual processing information is delivered to the data analysis module. In the data analysis module, the defect process and causes of the defect are analyzed and the analysis results are stored in the cloud. The cumulative past production data are utilized in a new product design of a similar product line to give feedback to designers about the defect rate, defect causes and ratio of past products for each process.

Flexible manufacturing can be achieved as the production schedule is changed according to production information on the product whenever the processes are performed in contrast to the traditional manufacturing, where processes are performed according to the pre-determined production schedule.

\subsection{Data model structure and database}

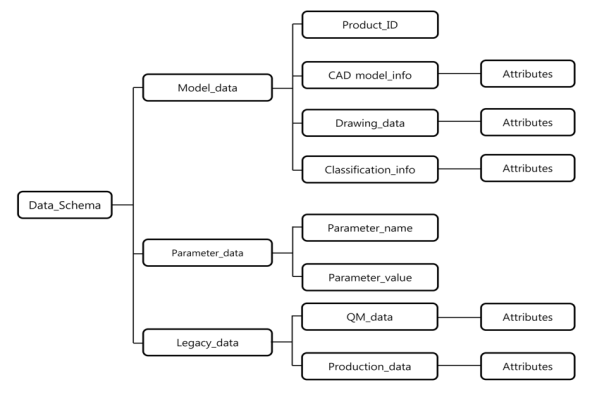

Fig. 2. Data schema of product-manufacturing integrations

Fig. 2 shows data schema of product-manufacturing integrations. It contains basic information on the product, such as the product no. (product ID), location of the CAD file, drawing information and product classification, and the parameter information contains design parameters and parameter values used in CAD modelling. In addition, data on defects, such as the presence of defects, defect causes and defect processes that occurred during the production phase, are added with regard to products in past production. The product data stored in the cloud (or database) are collected constantly in 
the design and production phases and transferred to the cyber model construction module whenever design verification is requested.

\subsection{Cyber model construction module}

The cyber model construction module plays a role in extracting design data, such as parameters from the CAD model of a new product and creating and transferring integrated data. Product ID, product classification information and parameter information, which are set in the CAD model, are verified in the cyber model construction module. The module also includes an extraction process of matching values in the CAD model. The integrated model data, including a matched value with each of the information names, are automatically created, and the created integrated model data are transferred to the database.

\subsection{CAD model analysis module}

The CAD model analysis module receives design data for past and new products from the cloud and predicts a design. The past and new product design data are compared based on the production data and quality management data of past manufactured products, thereby predicting expected problems or the defect rate during production.

\subsection{Defect alert module}

The defect alert module displays the design variables, and defects are predicted in the $\mathrm{CAD}$ model analysis module in the CAD model run by the CAD program. Furthermore, defect rates, parameters and its values with which defects are predicted are displayed on the dashboard in real time so that designers can easily utilize the product design feedback from manufacturing phase.

\section{$4 \quad$ Implementation}

Bottles of cosmetics from a Korean company were employed as a verification target of the study. A product is designed by utilizing the designer dashboard based on the past production data in a similar product line followed by the manufacturing phase in the factory. An RFID is attached to each of the products and facilities. The facility tags the ID of the RFID card attached to the product for communication between the facility and product. Once the facility reads the product ID, manufacturing information on the product (input solution, input amount, and processing temperature etc.) is read from the cloud, and a process is performed according to the manufacturing information. Once the process is complete, the facility collects data (actual input solution, actual input amount, and actual processing temperature) performed in the actual process and transfers the information to the cloud. If a defect occurs during the process, the defect information is delivered to the data analysis module, in which defect occurrence processes and causes are analyzed. The analyzed data are delivered to the cloud, and the data are 
utilized to provide feedback on the expected defective part in the design screen using a dashboard form when similar products are manufactured next. The design dashboard displays a defect rate per process, defect causes and ratio and analyses 3D CAD files of the newly designed product, thereby displaying the part where the defect is expected by highlighting it.

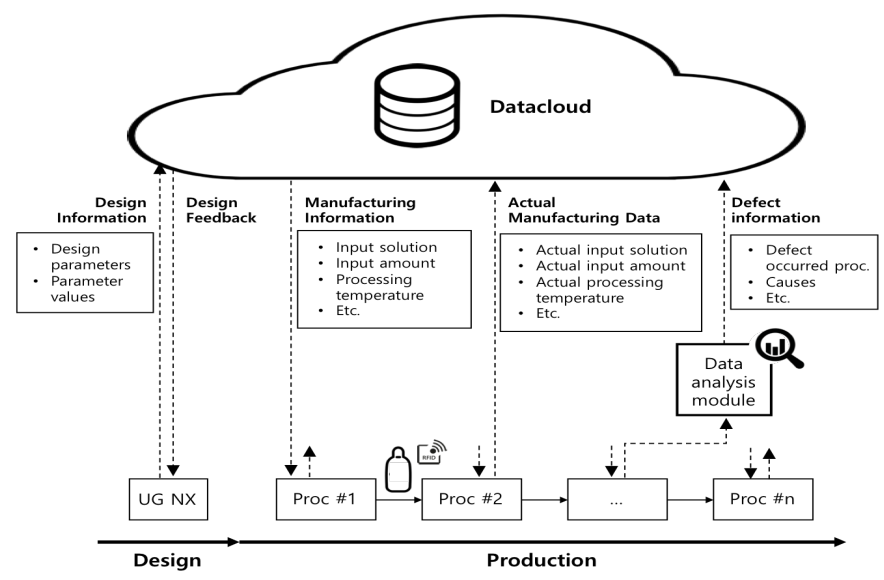

Fig. 3. Virtual smart factory based on real cosmetic factory

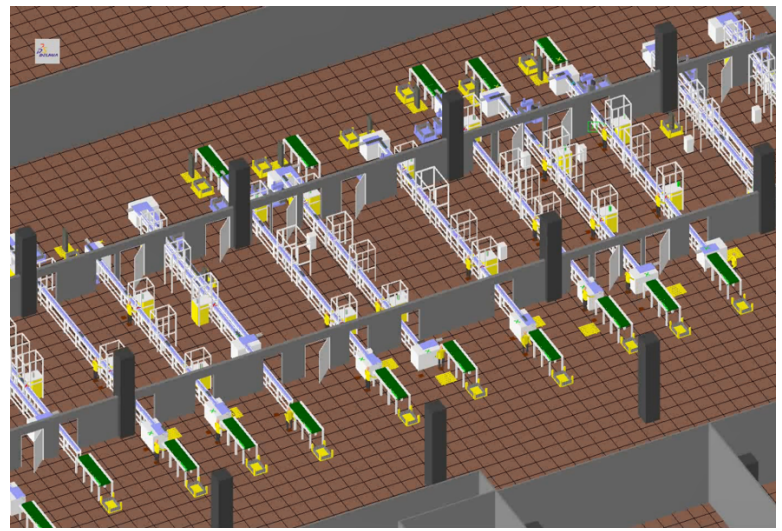

Fig. 4. Scenario of implementation

Assuming that the environment was a smart factory where a real-time data collection system was applied, the testbed was constructed to verify the concept based on scenarios. To prove the concept and need for the product CPS proposed in the present study, the proof of concept (POC) was conducted with cosmetics products (lotion, balancer and cream) and filling and assembly processes in a Korean company.

Since the POC could not be performed at a factory where CPS was implemented perfectly, the testbed was constructed by linking a physical testbed that replaces the 
actual processes with a virtual factory implemented by QUEST simulation. In the physical testbed, the RFID attached to the product is tagged to the RFID reader of the process facilities and the information of the product is read. Virtual testbed was implemented through the QUEST, which simulated a real factory as shown in Fig. 4. The study employed an RFID reader and Mifare1K Card from Advanced Card Systems.

For CAD software, NX 7.5 was used. First, a CAD file of the target product is fetched from the design support system. The design parameters and values in the model inputted through the CAD analysis module are extracted. Once the data extraction of the CAD model is complete, the past design data collected previously are fetched, and then the data of the newly designed CAD model and old data are compared. After each piece of design information is inputted into the cyber model construction module, the extracted data and related data of the past product data are matched, and the defect rate is predicted through the matched information. If the analysis prediction result is available, the defect rate is displayed on the dashboard through the defect alert module, and the predicted defect location is visualized by highlighting in NX. The manufacturing starts after re-designing to complete a design without the predicted defect.

Facilities in the filling process and lid assembly process which are the target processes, are displayed in the testbed as shown in Fig. 5, and the products are moved along the conveyor at a lot-level. Here, when the RFID reader attached to the facility tags the RFID card in the product, QUEST responds by running the production simulation.

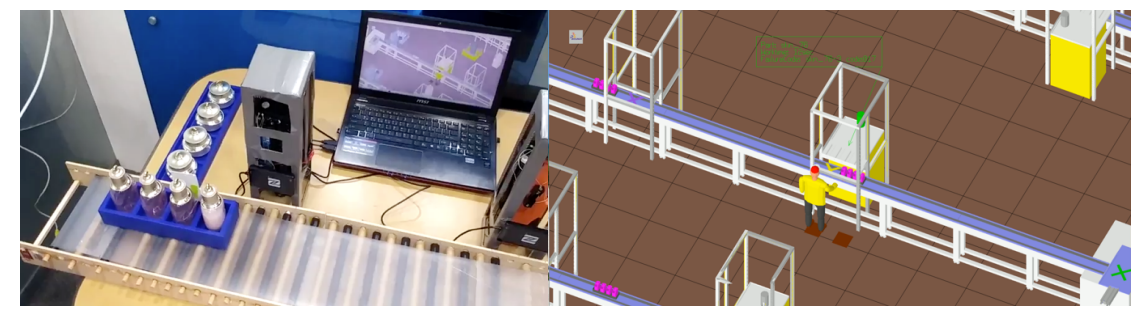

Fig. 5. Physical testbed with cosmetic products, RFID tags and readers and virtual testbed implemented by QUEST

Data collected during production simulation is stored in the database for use in the next product design. When a designer inputs a newly designed CAD model file, the CAD model analysis module is executed to extract the data in the corresponding model. Cyber model construction is the key activity that the overall Virtual testbed implemented by QUEST defect rate is generated through product data analysis in the same product line based on the new product data, and parameters where the defect is predicted are provided. Defect prediction results, in which the parameter variables where an ab-normality occurs, predicted defect code and cause of the defect are displayed on the dashboard once the part that corresponds to the parameter where the defect is predicted is selected on NX screen. Using the displayed information, the designer can understand and improve the design more intuitively by comparing the current design parameters and defect rates and ones changed. 


\section{Conclusion}

The present study proposed a framework by which smart manufacturing can be implemented utilizing big data collected via the IoT during manufacturing and verified the study content through a case study on a cosmetics company in Korea. However, it has a limitation regarding data items since it employs the manufacturing information of the virtual smart factory.

In future research, user's use history which is gathered by smart product itself, may be employed to product design phase to reflect user needs. So far, it has been hard to gather accurate use history from user since qualitative or indirect methods were used for investigation of user experiences. Big data analytics based on real use history is quantitative and direct method, so it may offer a new point of view in product development.

On the other hand, extended data will be employed to increase the accuracy of the analysis results and a study on new product development other than similar product lines will be conducted through trend prediction using social network and factor analysis.

\section{References}

1. Lee, Kyuteak., Lee, GunJae., Song, Byunghoon.: KEIT PD Issue Report; Trend of Smart Factory Technology. Korea Evaluation Institute of Industrial Technology, Cheonan (2015)

2. Hyundai Research Institute.: Upgrading Manufacturing Industry U.S.Japan·Germany Manufacturing Industry R\&D policy trend and Implication. Hyundai Research Institute, Seoul (2014)

3. Sha, L., Gopalakrishnan, S., Liu, X., Wang, Q.: Cyber-Physical Systems: A New Frontier. In: Machine Learning in Cyber Trust, pp. 3-13. Springer, Miami (2009)

4. Anderl, Reiner.: Smart Engineering for Smart Product. In: Smart Product Engineering, pp. 1-10. Springer, Heidelberg (2013)

5. Shin, Dongmin., Jeong, Bongjoo., Bo, Johyun.: Smart Manufacturing. $1^{\text {st }}$ edn. Epress, Seoul (2017)

6. Urtnasan, Erdenebayar., Jeon, Yu-Yong., Park, Gyu-Seok., Song, Young-Rok., Lee, SangMin.: A Study on Design Parameters for Ready-made Ear Shell of Hearing Aids. The Korean Institute of Electrical Engineers 60(5), pp. 1055-1061 (2011)

7. Harvard Business Review, https://hbr.org/2014/11/how-smart-connected-products-aretransforming-competition, last accessed 2018/04/06.

8. Cognizant.: Designing for Manufacturing's 'Internet of Things'. Cognizant, US (2014)

9. Van Eck, Maikel L., Sidorova, Natalia., Van der Aalst, Wil M.P.: Enabling Process Mining on Sensor Data from Smart Products. In: 2016 IEEE Tenth International Conference on Research Challenges in Information Science (RCIS), pp. 1-12 (2016)

10. Kang, Seongjoo., Jun, Jaeho., Kim, Hyunsoo., Jun, Ingul.: A Research on Design-Manufacturing-Service Interoperation Architecture for Managing Lifecycle of Smart-Connected Products. In: Korean Institute of Information Scientists and Engineers, pp. 638-641 (2015)

11. Han, Chaejong.: Constitution and Principle of RFID System. $1^{\text {st }}$ Edn. Micros oftware, Seoul (2006) 